\author{
MIRA KADRIC \\ Universität Wien
}

\title{
Strategien einer Übersetzungsdidaktik
}

ABSTRACT. The present article is devoted to discussing and carrying out translation methodology classes on German, Bosnian, Croatian and Serbian. The author assumes that not only the classes but also their planning itself should be perceived as a process.

Für die Gesamtplanung einer Lehrveranstaltung bilden die didaktischen Grundfragen nach dem Wozu, dem Was, dem Wie und Womit des Lehrens und Lernens den Ausgangspunkt der Konzeption. Die Grundfragen betreffen also die Ziele, Inhalte, Methoden und Hilfsmittel des Unterrichts und schließen mit der Frage nach dem Erfolg des Lehrens und Lernens, der sich sowohl in der Beurteilung der studentischen Leistungen (Prüfung) als auch in der Bewertung der Lehrleistungen (Evaluation der Lehrveranstaltung) manifestiert. Auch diese abschließenden Fragestellungen erfordern eine Auseinandersetzung mit Kriterien und Modi der Leistungsbeurteilung. Im folgenden Beitrag wird die Abhaltung einer Lehrveranstaltung zur translatorischen Methodik im Sprachenpaar Deutsch-Bosnisch/Kroatisch/Serbisch ${ }^{1}$ beschrieben sowie die Problematik einer solchen Lehreinheit diskutiert. Den Ausgangspunkt der Diskussion bildet die Behauptung, dass starre Planungskonzepte in der modernen Unterrichtspraxis - insbesondere bei Übungen - didaktisch nicht sinnvoll sind. Daher wird nicht nur die Lehrveranstaltung selbst, sondern auch ihre Konzeption als Prozess angesehen. Daraus folgt, dass es lediglich eine Grobplanung geben kann die eine Öffnung für eventuelle Korrekturen des Lehr- und Lerngeschehens aufgrund laufender Überprüfungen der Lehrwirksamkeit durch Rückmeldungen und Zwischenarbeit erlaubt.

${ }^{1}$ Die ehemals serbokroatische Sprache wurde am Wiener Institut für Übersetzen und Dolmetschen ohne Änderung bzw. Anpassung des Lehrplans in "Bosnisch/Kroatisch/Serbisch" umbenannt. Die Lehrenden sind hier gefordert, im Rahmen ihres Unterrichts auch die Auseinanderentwicklung dieser vormals sehr ähnlichen Sprachvarietäten zu berücksichtigen. 


\section{PLANUNG DER LEHRVERANSTALTUNG}

Bei der Planung von Lehrveranstaltungen spielen nicht nur didaktische Überlegungen eine Rolle, sondern auch die organisatorischen Rahmenbedingungen. Im Rahmen des Studiums "Übersetzen und Dolmetschen" am Wiener Zentrum für Translationswissenschaft werden Übersetzungsübungen in der Regel ab dem 3. Semester ${ }^{2}$ empfohlen, so auch im Sprachenpaar Deutsch-Bosnisch/Kroatisch/Serbisch. Das Studium insgesamt und die kleinen Sprachen insbesondere sind so konzipiert, dass die meisten Übungen für Studierende aller Semester in Form von Wahlpflichtlehrveranstaltungen angeboten werden. Konkret heißt das, dass dieselbe Übung Studierende des ersten Abschnitts (beispielsweise im 3. Semester) mit Studierenden des zweiten Studienabschnitts ( beispielsweise im 9. Semester) absolvieren. Daraus folgt, dass die Planung einschlägiger Lehrveranstaltungen nicht nur eine Reihe von Entscheidungen über didaktische Gestaltungselemente, sondern auch eines gewissen Maßes an Kreativität und Flexibilität bedarf. Eine Auswahl von Zielen, Inhalten und Methoden ist so zu treffen, dass ein möglichst großer Lerneffekt bei allen "Studierendenschichten" erzielt werden kann. Diese vorgegebenen Rahmenbedingungen erlauben kaum eine frühe Detailplanung der gesamten Lehrveranstaltung. Die Detailplanung kann also nicht isoliert stattfinden, sondern muss im Austausch mit den Studierenden erfolgen. Die Einbeziehung der Studierenden in die Planung hat sich in der Praxis als vorteilhaft erwiesen.

Den Kern der Planung und Gestaltung der Lehrveranstaltung bildet die Lernzielbestimmung. Diese ist angesichts der Struktur der Zielgruppe äuBerst schwierig: Wie bereits erwähnt, können angesichts der Heterogenität der Gruppe im voraus lediglich die Groblernziele festgelegt werden. Diese orientieren sich nach den allgemeinen Fachlernzielen der Studienrichtung. Maßgeblich sind dabei drei Determinanten der Ausbildung: die theoretische Fundierung, die Integration von Theorie und Praxis sowie das Textverständnis und die Textproduktion als besonderer Schwerpunkt. Mit diesen Schwerpunkten wird eine funktionalistische Lernform mit theoretischer Reflexion angestrebt. Darüber hinaus gehören meines Erachtens, z. B. die Auswahl von Inhalten auch Fragestellungen nach der Priorität oder Reduktion der Inhalte oder inhaltliche Querverbindungen zu anderen Lehrveran-

2 Das beschriebene Studium ist in zwei Studienabschnitte unterteilt. Es gibt nur einige wenige Lehrveranstaltungen, die in einem bestimmten Studienabschnitt absolviert werden müssen; vielmehr ist es den Studierenden überlassen, zu welchem Zeitpunkt sie bestimmte Lehrveranstaltungen besuchen. Nicht selten aber muss aus organisatorischen Gründen die gleiche Lehrveranstaltung mehrere Semester lang besucht werden, wodurch häufig Studienanfänger mit Studierenden höherer Semester dieselbe Lehreinheit besuchen. 
staltungen sowie die Berücksichtigung studentischen Vorwissens, und der Anregungen und Vorstellungen der Studierenden, um nicht nur eine möglichst nützliche Gestaltung der Lehrveranstaltung vorzunehmen, sondern auch die Motivation ${ }^{3}$ der Studierenden aufrecht zu erhalten. Wie Wilss treffend feststellt, spielt die Motivation im Lernfortschritt eine bedeutende Rolle:

Wenn ein Student motiviert ist (oder motiviert werden kann), (...) und wenn taugliche Methoden für die Steuerung des übersetzerischen Lernprozesses zur Verfügung stehen (oder die Bereitschaft besteht, diese im Rahmen des Möglichen zu entwickeln), kann ein Student eine bemerkenswert hohe Festigkeitsebene erreichen, auch wenn zu Beginn des Studiums deutliche Unterschiede in den individuellen Ausgangspositionen festzustellen waren. (Wilss 1992: 59)

\section{DURCHFÜHRUNG DER LEHRVERANSTALTUNG: RAHMENBEDINGUNGEN UND DIDAKTISCHE ELEMENTE}

Entsprechend der Lehrveranstaltungsform (Übung) muss der Unterrichtsverlauf so strukturiert werden, dass Studierende unterschiedlichen Wissensstands vom Unterricht profitieren bzw. die angestrebten Lernziele erreichen können. Dazu sind die Fragen nach der Auswahl geeigneter Methoden entscheidend. Als eine der ersten Fragen stellt sich jene nach den Aktivitäten (sowohl seitens der Lehrenden als auch der Studierenden), die gesetzt werden müssen, um zu den gesteckten Zielen gelangen zu können. Im Rahmen der beschriebenen Übung werden zwei Kategorien von Aktivitäten unterschieden: Elemente, bei denen die Hauptaktivität bei der Lehrenden liegt und solche, bei denen die Hauptaktivität die Studierenden übernehmen. Lehrerzentrierte didaktische Elemente würden folgende Arrangements umfassen:

- Eröffnen des Unterrichts/Einstieg/Vorstellung des LV-Konzepts,

- ev. Vorträge,

- Problematisierung, Strukturierung,

- Informationsvermittlung bzw. -erarbeitung,

- Arbeitsauftrag,

- Fragen beantworten,

- Zusammenfassen,

Studierendenzentrierte didaktische Elemente betreffen

- Analyse des Arbeitsauftrags durch Diskussion, Erfahrungsaustausch, Argumentation ,

3 Untersuchungsergebnisse in Verbindung mit den mentalen Denkprozessen haben etwa ergeben, dass Sympathie bzw. Antipathie zu Textinhalten die Übersetzung beeinflussen (vgl. dazu Hönig 1995). 
- Herstellen von Zusammenhängen,

- Übertragung auf andere Situationen, Umsetzung in Handlungsstrategien,

- Recherchieren,

- Präsentation von Ergebnissen,

- Produktherstellung,

- Reflexion über durchgeführte Arbeiten.

Da die Qualität von Lernprozessen stark vom Ausmaß der Aktivität des Einzelnen abhängt, muss nach Möglichkeiten gesucht werden, die anteilsmäßigen Aktivitäten der Studierenden entsprechend ihrem Leistungsniveau optimal einzusetzen. Es hat sich gezeigt, dass eine gute Möglichkeit darin besteht, im Laufe des Semesters verschiedene Interaktionsformen einzusetzen, nämlich sowohl Einzelarbeit als auch Tandem- und Gruppenarbeit und Plenum.

Die einzelnen Lernschritte werden dem Lehrveranstaltungstyp angepasst, d.h. die Abfolge von möglichen Lernschritten richtet sich nach dem Motto "Aufnahme - Verarbeitung - Wiedergabe". Ziel der Lehrenden in dieser Übung ist es, Aufträge, wie sie in der Praxis an die Lehrende herangetragen werden, im Unterricht $z u$ simulieren. Das beinhaltet alle Aspekte einer Auftragsvergabe. In einem Drei-Schritt-Schema kann diese Abfolge wie folgt dargestellt werden:

- Vorbereitungsphase: Anfrage des Auftraggebers, Angeboterstellung, Verhandlungen mit dem Auftraggeber,

- Durchführungsphase: Diskutieren, Recherchieren, Rückfragen an den Auftraggeber, Textproduktion, Textredaktion, Layout,

- Nachbereitungsphase: Abgabe, abschließende Korrespondenz, Rückfragen des Auftraggebers.

Grundsätzlich erfolgt der Einstieg in die Lehrveranstaltung über einen thematischen Aufhänger. Es geht dabei um eine geeignete theoretische Darbietung, die motivationsträchtige Inhalte enthält, eventuell ergänzt durch praktische Beispiele, die anschließend mit den Teilnehmerinnen diskutiert werden. Als Ausgangspunkt dienen dabei die methodischen und didaktischen Erkenntnisse der funktionalen Translationstheorie. ( $\mathrm{Zu}$ diesem Zeitpunkt sollten alle Studierenden eine zweisemestrige, jeweils als zweistündige Pflichtvorlesung angelegte "Einführung in die Translationswissenschaft" absolviert haben. Diese Lehrveranstaltung vermittelt die wissenschaftlichen und berufskundlichen Grundlagen des Studiums; das heißt, die zentralen Begriffe der modernen Translationswissenschaft sowie translationsrelevante Aspekte der Sprachwissenschaft können als bekannt vorausgesetzt werden bei Bedarf werden diese Begriffe und ihre Inhalte jedoch noch einmal gemeinsam erörtert.). Der in der Übung zur translatorischen Methodik ange- 
wendete theoretische Aufhänger dient zur Einarbeitung in das jeweilige Thema und die eigenständige, kritische Auseinandersetzung mit dem in der Übung behandelten Übersetzungsauftrag.

Die Texte werden in Form eines Übersetzungsauftrages definiert und bearbeitet. Ausgehend von einer detaillierten Auftragsanalyse werden in einer studierendenzentrierten Diskussion - die Lehrende übernimmt hier im Idealfall lediglich die Rolle der Diskussionsleiterin - zunächst die für eine Angeboterstellung relevanten Fragen geklärt und Vorschläge gemacht, welche Strategien bei den Verhandlungen mit dem Auftraggeber zu wählen sind. In einem Sprachenpaar wie Deutsch-Bosnisch/Kroatisch/Serbisch ergeben sich insbesondere in gegebener politischer Situation eine Reihe von Fragen, die vor der Annahme eines Auftrags geklärt werden müssen. In dieser Phase der Auftragsbearbeitung reihen sich auch konstruktive Diskussionen zur translatorischen Ethik. Die Aufgabe der Lehrenden - als notwendige theoretische Vorinformation an die Studierenden - kann in dieser Situation darin bestehen, einen Impulsvortrag zur Verantwortung der Translatorin im Sinne einer Rollen- und Aufgabenverantwortlichkeit zu geben, nämlich aufzuzeigen, wie die umfassende translatorische Tätigkeit zu realisieren ist und welche ethischen Forderungen an die Translatorin $\mathrm{zu}$ stellen sind bzw. nach welchen Kriterien die moralische Verantwortung der Translatorin einzustufen ist. Der Ruf nach Ethik als Forderung nach einem übergeordneten Regelwerk, das vorgibt, was moralisch verboten, geboten oder erlaubt sein soll, ist ein fester Bestandteil der Übersetzungsdidaktik. Die übersetzerische Ethik, welche die Gesamtheit sittlicher Normen und Maximen, die der verantwortungsbewussten Einstellung zum Beruf zugrunde liegen, bildet, umfasst sowohl Normen und Vorschriften als auch Ehrenkodizes. Die Studierenden werden mit diesem Regelwerk bekannt gemacht, wobei anschließend die Fragestellung nach der etnischen Berechtigung der translatorischen Handlungen im Hinblick auf das Gesamtziel mit allgemeiner Gültigkeit diskutiert wird. Grundsätzlich werden diese Prinzipien zunächst als tradierte Werte, zeitlos und kulturneutral vorgestellt und als innere Dispositionen der Translatorin, als erworbene moralische Kompetenz, die die Gesinnung bzw. das Gewissen der Translatorin aus dem Raum der Willkür und moralischer Überzeugungen in einen argumentativen Rahmen stellt, verstanden.

An die Einheit zu allgemeinen Fragestellungen der translatorischen Verantwortlichkeit schließt eine studierendenzentrierte Diskussion im Plenum an, wobei die allgemeinen Fragestellungen zur Ethik unmittelbar situationsaktiviert werden. Diskussionen ${ }^{4}$ in dieser Vorbereitungsphase der simulier-

${ }^{4}$ Die ethische Komponente wurde hier lediglich exemplarisch angeführt; selbstverständlich gibt es in dieser Phase eine ganze Reihe anderer diskussionswerter Themen die auftragsbedingt sind. 
ten Auftragsvergabe beeinflussen in der Regel die gesamte spätere Arbeit am Text.

In der anschließenden Durchführungsphase soll aufbauend auf den in der Vorbereitungsarbeit erworbenen Kenntnissen die Fähigkeit ausgebaut werden, in Form von Textarbeit kritisch mit Texten umzugehen und Textproduktion zu üben. Eine der Maximen dieser Übung besteht darin, dass die Studierenden selbständig die Antwort auf eine Frage bzw. die Lösung für ein Problem finden sollen, ohne dass dafür eindeutige Antworten oder Lösungsmuster vorliegen. Das führt dazu - da die Aktivität bei der Durchführung der Arbeit vorwiegend bei den Studierenden liegen sollte -, dass die Lehrende im aktuellen Prozess in den Hintergrund tritt. Dies erfordert jedoch eine Grobplanung, die unterschiedliche Faktoren und Eventualitäten berücksichtigt, wie man sich als Lehrende doch Einflussmöglichkeiten offen lässt. Denn während der Arbeit können nur noch kleinere Korrekturen vorgenommen und minimale Wissenslücken ausgeglichen werden, wenn der Sinn der selbständigen Arbeit und die größtmögliche Eigenaktivität der Teilnehmerinnen gewahrt werden soll. Daher sind genaue Festlegungen der Arbeitsaufträge zu treffen, die in der detaillierten Definition der Ziele und Reihung ihrer Wertigkeit bestehen, die beispielsweise folgende Punkte enthalten kann:

- Einteilung (Einzel-, Tandem-, Gruppenarbeit),

- Modus der Arbeitsverteilung,

- Exakte, möglichst eindeutige Beschreibung der Aufgabe,

- Alternativvarianten für den Fall der Über- oder Unterforderung bzw. Ausfall von Teilnehmerinnen,

- Orientierungshilfen zur Arbeitsweise,

- Angaben zur Dokumentation und Präsentation der Arbeit,

- Angaben zum Zeitrahmen,

- Angaben zum Ort und/oder Form für die selbständige Arbeit bzw. ev. Möglichkeiten, andere Räume zu benützen (z. B. Bibliothek),

- Kriterien, nach denen beurteilt werden kann, ob und mit welcher Qualität die Ziele dieser Arbeit erreicht worden ist.

Diese Einteilung ist natürlich nicht nach einem starrem Muster zu vollziehen, vielmehr wird sie sich erst in der Detailplanung der Lehrveranstaltung ergeben. Denn sowohl für die Definition als auch für die Beschreibung des Arbeitsauftrages müsste die Lehrende die Vorkenntnisse und sonstigen vorausgesetzen Kompetenzen der Studierenden genau kennen oder nach der Dauer der bereits absolvierten Studienzeit einschätzen können. Selbstverständlich besteht hierbei die reale Gefahr, dass es zu Über- oder Unterschätzungen kommt. Erfahrungsgemäß empfiehlt sich aus diesem Grund eine abwechslungsreiche Organisation der Lernübung. Das kann auf unter- 
schiedliche Weise erreicht werden: Falls der Arbeitsauftrag an Kleingruppen vergeben wird, können eine der Sachlage entsprechende und nach verschiedenen Kriterien homogene oder heterogene Zusammensetzung der Gruppen überlegt sowie Kriterien und Vorgangsweisen für die Gruppenbildung bestimmt werden.

Die Arbeitsweise wird ebenfalls festgelegt; zumindest werden aber Hinweise ausgearbeitet, wie eine passende Arbeitsweise im Hinblick auf den Inhalt und Prozess für eine Aufgabe herauszufinden ist. Dabei wird auch der Zeitaspekt berücksichtigt: Zeitangaben sind auch deshalb wichtig, weil die Studierenden von Beginn der selbständigen Arbeit an mit zeitlichen Vorgaben konfrontiert sind und den Umgang damit lernen müssen.

Die voranstehenden Punkte werden im Plenum geklärt. Anschließend wird Zeit für Fragen gelassen. Wenn der Arbeitsauftrag erteilt ist und etwaige Unklarheiten bereinigt sowie die erforderlichen Materialien verteilt sind, beginnt die studierendenzentrierte Arbeit in der Lehrveranstaltung. Die weitere Aufgabe der Lehrenden ist es im Idealfall, die selbständig arbeitenden Gruppen rotierend zu kontaktieren und ihnen beratend zur Seite zu stehen.

Es wurde bereits gesagt, dass die Arbeit unter genauer Angabe des Übersetzungsauftrags erfolgt. Dabei wird der Auftrag explizit formuliert, wobei insbesondere folgende Angaben zum Ausgangstext und Zieltext berücksichtigt werden:

- Ausgangsbezogene Daten: Produzent/Sender, Adressat, Zeit, Ort, Medium und Kommunikationsanlass,

- Zieltextbezogene Daten: Zweck der Übersetzung, Adressaten, Zeit, Ort, Medium und Zieltextverwendung.

Aus diesen Vorgaben ergeben sich zunächst allgemeine Fragestellungen, die didaktisch im Zusammenspiel der sogenannten "W-Fragen" 5 erörtert werden können. Die Gruppenarbeit besteht also zunächst in der Klärung dieser Fragen. Als didaktisch sehr fruchtbare Einheit eignet sich dafür die Diskussion. Hierbei können die Studierenden ihr Verständnis des Auftrags erproben, festlegen oder nötigenfalls korrigieren. Durch Erfahrungsaustausch werden individuelle Anknüpfungspunkte für die systematische Behandlung des Auftrags geschaffen. Dabei können die unterschiedlichsten Sichtweisen ein und desselben Sachverhalts miteinander konfrontiert und

5 Diese von Harold D. Lasswell (1948) entwickelte und auch in der Translationswissenschaft (vgl. Reiß 1984a, Hönig 1986) viel verwendete Fragenkette wurde für didaktische Zwecke von Nord (1991) erweitert bzw. modifiziert. Sie lautet: Wer übermittelt wozu wem über welches Medium wo wann warum einen Text mit welcher Funktion? Worüber sagt er was (was nicht) in welcher Reihenfolge unter Einsatz welcher nonverbalen Elemente in welchen Worten in was für Sätzen in welchem Ton mit welcher Wirkung? 
abgewogen werden, Vorgangsweisen festgelegt und Bewältigungsstrategien und Lösungsvorschläge entwickelt werden. Dabei wird nicht nur die sachliche Kompetenz weiterentwickelt; hier kommt auch die für die translatorische Tätigkeit wichtige soziale bzw. kommunikative Komponente zur Geltung.

Als Ergebnis der Diskussion ergibt sich in der Regel eine Aufstellung der notwendigen Arbeitsschritte sowie eine gruppeninterne Arbeitsteilung. Diese betrifft die Recherchiermöglichkeiten bzw. eine Liste der für die Erfüllung des Arbeitsauftrages erforderlichen Materialien, Unterlagen oder sonstigen Hilfsmittel. In diesem Prozess der Findung, Auswertung und Klassifizierung der Hilfsmittel spielt das Primat der Kooperation ${ }^{6}$ eine entscheidende Rolle. In einer konstruktiven Diskussion können Studierende ihre Meinung eigenständig einbringen und Wissen nicht nur reproduzieren, sondern aktiv herstellen, indem sie Aufgaben übernehmen, die alte und neue Informationen vernetzen. Der individuelle Beitrag zu einem gemeinsamen Lernziel führt erfahrungsgemäß zu Kreativität und Vertrauen in die eigene Leistung, womit die Motivation sowohl des Einzelnen als auch der Gruppe insgesamt steigt. Diese Arbeitsform kann auch einer heterogenen Gruppe zu guten Ergebnissen verhelfen.

Der anschließenden Textproduktionsphase geht eine detaillierte Textanalyse voraus: Es werden zunächst die für die Zieltextproduktion relevanten Fragen systematisch geklärt: Übersetzungstyp, Textsorte, Textfunktion, Medium und Zeitbezug. Die Beschäftigung mit Texten stellt insgesamt einen zentralen Punkt der Studienrichtung dar. Darunter wird vorwiegend Textarbeit in Form von Textverstehen, Textanalysieren und Textproduzieren verstanden. Methodisch gesehen erfolgt die Textverstehensarbeit inhaltsanalytisch, indem die verborgenen Implikate untersucht werden und in der Textproduktionsphase Prozesse wie Auswählen, Organisieren und Formulieren in den Text transformiert werden. Eines der wichtigsten Instrumente in der Textproduktion ist sicherlich die Beschaffung externer Informationen, die, wie bereits beschrieben, in der Vorbereitungsphase des Übersetzungsauftrags erfolgt. Die Vorbereitung geschieht also, indem nach relevanten Informationen, relevanten (Parallel)Texten gesucht wird, aber auch indem die Ergebnisse des Einlesens in unterschiedlicher Weise verwertet werden. Der Textproduktionsprozess wird von drei Faktoren beeinflusst: adressatengemäße, sprachliche Form des Textes, inhaltlich-thematische Orientierung des Textes und Wissensgehalt des Textes. Jeder dieser Faktoren bedingt eine schwerpunktmäßige Auseinandersetzung mit der konkreten Problematik. Es gibt kaum eine Lehrveranstaltung zur Translatorik, in der lediglich die translatorisch-technischen Fragestellungen auf dem Programm

${ }^{6}$ Der professionellen Translation - die in der Übung simuliert wird - liegt Arbeitsteiligkeit und Kooperativität per definitionem zugrunde (vgl. Holz-Mänttäri 1984). 
stehen; vielmehr erfolgt meist gleichzeitig eine sprachliche Übung, eine Wissensvermittlung usw. So auch in der beschriebenen Übung.

Die äußere, sprachliche Form des Textes erfordert die einwandfreie Beherrschung der Mutter- bzw. Fremdsprache. Diese notwendige Voraussetzung der Bi- bzw. Multilingualität und -kulturalität ist während der Studienzeit in der Regel nicht gegeben und wird erst im Studium erworben. Die Übungen - oft unabhängig davon, wie sie definiert werden - sind ein $\mathrm{Zu}$ sammenspiel zwischen dem Erwerb der Sprach-, Text- und translatorischen Kompetenz. ${ }^{7}$

In der beschriebenen Lehrveranstaltung hängt der Erfolg des Erwerbs dieser Kompetenzen eng mit der Studierendengruppe zusammen: diese setzt sich vorwiegend aus den Angehörigen der Sprachminderheiten zusammen, deren (häufig vermeintliche) Bilingualität ${ }^{8}$ sie zur translatorischen Berufsausbildung verleitet. Daher ergibt sich die Arbeit am Ausbau der Sprachkompetenz als notwendiger, integraler Teil der Übung. Die Arbeit umfasst in der Regel sowohl die mutter- als auch fremdsprachliche Kompetenz. Am Beispiel des Bosnischen/Kroatischen/Serbischen bedeutet dies - im Hinblick auf die Zusammensetzung der Gruppe aus Personen, die aus politischen Gründen (zu)gewandert sind - die Verfolgung der neuesten Entwicklungen dieser Sprachvarietäten, die nun offiziell selbständige Sprachen sind, und sich tatsächlich rasant auseinander entwickeln. Die Auseinandersetzung mit dieser Problematik erfolgt je nach Erforderlichkeit, aus dem gerade bearbeitenden Thema ausgelagert oder aber im Zuge der konkreten Textarbeit (wobei hier die Grenze ohnehin fließend ist).

Die übersetzerische Textarbeit umfasst in der Phase der Rezeption verschiedene pragmatisch-funktionale Verstehensstrategien, die verschiedene textexterne Bedingungen sowie die Aufarbeitung terminologischer Rezeptionsschwierigkeiten berücksichtigen. Dabei wird explizit auf Textaufbau, Lexik, Syntax und multimediale Elemente des Textes eingegangen. Die sich ergebenden Fragestellungen werden in verschiedene Kategorien zusammengefasst: pragmatische Übersetzungsprobleme, kulturpaarspezifische Übersetzungsprobleme, sprachenpaarspezifische Probleme und textspezifische Probleme. ${ }^{9}$ Pragmatische Übersetzungsschwierigkeiten betreffen die Kommunikationssituation und das darin unterschiedlich gelagertes Vorwis-

${ }^{7}$ Im Rahmen dieses Beitrags wird nicht zwischen muttersprachlicher und fremdsprachlicher Kompetenz unterschieden. Zur Rolle der muttersprachlichen Kompetenz vgl. Resch (1998); zur Rolle der fremdsprachlichen Kompetenz Hansen (1998).

${ }^{8}$ Aufgrund verschiedener Faktoren weisen diese Studierenden häufig mangelnde Kompetenz sowohl in ihrer Muttersprache als auch in der Bildungssprache auf, womit ihre Sprachkompetenz eher als mehrfache Halbsprachigkeit bezeichnet werden könnte (zum Semilingualismus bei Migrantenkindern vgl. Skutnabb-Kangas 1981).

${ }^{9}$ Diese Kategorisierung geht auf Nord (1998: 352) zurück und wurde von ihr als Ausgangspunkt für einen leichteren Zugang zur Übersetzungstechnik im Unterricht vorgenommen. 
sen des Ausgangs- und Zielpublikums; kulturpaarspezifische Übersetzungsschwierigkeiten betreffen den Unterschied zwischen den ausgangs- und zielkulturellen expliziten und impliziten Normen und Konventionen; sprachpaarspezifische Übersetzungsschwierigkeiten ergeben sich aus dem Kontrast der ausgangssprachlichen und zielsprachlichen Strukturen; textspezifische Übersetzungsschwierigkeiten betreffen, individuelle kommunikative Vorkommen und können nicht verallgemeinert oder auf andere Texte angewendet werden. Mit dem Textverstehen ist die Recherche eng verbunden: wichtig für die Übung ist es zunächst, sich mit diesem Thema überhaupt zu konfrontieren. Es wird daher geübt, den nötigen Recherchebedarf zu erkennen, zwischen Reflex und Reflexion zu unterscheiden. ${ }^{10}$

Die Phase der produktiven Textarbeit kann nicht lediglich als Schreiben des Textes verstanden werden. Sie ist vielmehr ein interaktiver Prozess: Das Schreiben ist von kontinuierlicher Planung und Revision begleitet. Der Fokus der Übung liegt dabei im Erwerb bzw. in der Weiterentwicklung verschiedener, für die Translatorinnen unabdingbarer Textkompetenzen, wie kulturspezifisch geprägter Kommunikationsintentionen und ihre Widerspiegelung im Text: äußere Form, formaler Aufbau, Verbalisierungsmuster für Routineformulierungen, textsortenspezifische Sprachverwendung usw. Als ein häufig auftretendes Problem in der Textproduktion der bereits angesprochenen (vermeintlich) zweisprachigen Studierendengruppe ist die Interferenz. Es handelt sich dabei sowohl um die Textinterferenz, wobei rein sprachliche Normen verletzt werden, als auch um translatorische Interferenz, womit die intendierte kommunikative Wirkung nicht erreicht wird.

Die abschließenden Schritte des simulierten Übersetzungsauftrags beinhalten die Redaktion des Textes, die Anpassung der Typographie und des Layouts sowie die abschließende Korrespondenz mit dem Auftraggeber, einschließlich der Erstellung einer Honorarnote.

In einer Übung mit diesem Lernkatalog kann sich die gleichberechtigte Aufgabenverteilung der Gruppe etwas verschieben, wobei die fortgeschrittenen Studierenden unter Umständen die führende Rolle innerhalb der Gruppe gewinnen können (womit sich Anfänger unter Umständen weniger motiviert fühlen). In der beschriebenen Lehrveranstaltung wird versucht, durch häufiges Wechseln zwischen individueller, Tandem- und Gruppenarbeit die Arbeit abwechslungsreich zu gestalten und die angesprochene Problematik möglichst zu vermeiden. Ziel dieser Lehrveranstaltung ist es, dass Studierende durch diese Übung lernen, Ergebnisse zu präsentieren, über Recherche $\mathrm{zu}$ berichten, Aufgaben und Ziele $\mathrm{zu}$ formulieren und zu kritisieren sowie durch Diskussion und Argumentation zu kommunizieren und Entscheidungen zu begründen. Insgesamt sollte die Lehrveranstaltung einen

10 Vgl. dazu Hönig (1986). 
konstruktiven Prozess darstellen, indem Probleme bei gleichzeitiger Vermittlung des Einblicks in die berufliche Praxis eigenständig formuliert und gelöst werden. Auf ein vorhandenes Wissen und die Kenntnis konkreter Einzelheiten gestützt, soll Verstehen und Analyse von Elementen geübt werden, die sich in der Anwendung im Einzelfall widerspiegeln.

\section{BEWERTUNG DER LERN- BZW. LEHRLEISTUNG}

Selbstverständlich kann in einer Übung, an der eine heterogene Studierendengruppe teilnimmt und die mehrere Aufgaben zu erfüllen hat, nicht alles optimal gestaltet werden. Der tatsächliche Erfolg der Lehrveranstaltung zeigt sich in der Leistungsbewertung: sowohl in der Bewertung der Lehrleistung, der Evaluation, als auch in der Lernleistung, der Prüfung. In der Beurteilung der Lehrleistung hat sich die begleitende Evaluierung der Lehrveranstaltung (während des Semesters) und eine abschließende Evaluierung am Ende des Semesters als äußerst hilfreich erwiesen. Die begleitende Überprüfung wird einerseits implizit durch Beobachtung, Prozessanalyse und informelles Feedback durchgeführt; andererseits wird mehrmals ${ }^{11}$ im Semester eine Befragung, die inhaltlich auf diese konkrete Lehrveranstaltung zugeschnitten ist, durchgeführt. Am Ende des Semesters wird die von der Universität Wien vorgesehene Evaluierung durchgeführt: mittels Fragebogens mit eher allgemeinen Fragen zur Form und Abhaltung der Lehrveranstaltung bzw. zur lehrenden Person.

Die Beurteilung der studentischen Leistungen und der Kompetenznachweis in einer solchen Lehrveranstaltung ist im Hinblick auf den Charakter der Lernziele schwierig: Es soll sowohl der Bezug zur Berufspraxis als auch der Nachweis abstrakter Leistungsdimension erbracht werden. Der Nachweis dieser Kompetenzen ist situationsabhängig und bringt problematische Bewertungskriterien mit sich. Daher stellt sich die Frage, welcher Modus für die Leistungsüberprüfung am besten geeignet wäre. In der Übung zur translatorischen Methodik Deutsch-Bosnisch/Kroatisch/Serbisch wurden bereits verschiedene Modi angewendet. Aus der dreijährigen Analyse der Rückmelde- und Prüfungsergebnisse wurde die Schlussfolgerung gezogen, dass eine kontinuierliche Bewertungsform für diese Übung geeignet ist: Neben der internen Evidenz, die die Beobachtung der Lehrenden sowie detailreiche Rückmeldungen der Studierenden enthält, werden mehrere kleinere Arbeiten während des Semesters beurteilt. Es handelt sich dabei in der Regel um Tandem- oder Gruppenarbeit, eine individuell erstellte Arbeit und

" Hier werden keine strengen Regeln befolgt; vielmehr wird es von der konkreten Lernsituation und -gruppe abhängen, ob die Rückmeldung durch Diskussion oder einen schriftlichen Fragebogen erfolgt bzw. wie oft eine Rückmeldung stattfindet. 
eine kleinere Abschlussarbeit. Durch die Aufteilung auf mehrere kleinere Leistungsnachweise wird der Versuch unternommen, die Nachteile einer klassischen Prüfungssituation, wie Einfluss von Situationsfaktoren und geringe Entfaltungsmöglichkeiten, $\mathrm{zu}$ vermeiden.

\section{SCHLUSSBEMERKUNGEN}

Bei der Planung und Durchführung der Lehrveranstaltung muss insgesamt berücksichtigt werden, dass nicht alle Bereiche des Lehr- und Lerngeschehens tatsächlich erfasst werden können. Daher sollte die nähere Bestimmung des Lernziels nicht außer acht gelassen werden: Diese Übersetzungsübung versteht sich als Simulation echter Übersetzungssituationen, d.h. die Studierenden sammeln über die abstrakte Betrachtung praktische Erfahrungen, wobei sie die verschiedenen Arbeitssituationen selbst erleben und anschließend reflektieren. Dabei zeigt sich, dass Lernen umso besser funktioniert, je aktiver die Lernenden dabei sind. Die Lehrende setzt dabei geeignete Handlungsrahmen, in denen die Studierenden kreativ werden können.

\section{BIBLIOGRAPHIE}

Eigler, Gunther, 1998. Textkommunikation als Wechselspiel von Textverarbeiten und Textproduzieren. In: Unterrichtswissenschaft. Zeitschrift für Lernforschung, 51-67.

Hansen, Gyde., 1998. Die Rolle der fremdsprachlichen Kompetenz. In: Snell-Hornby, Mary/Hönig Hans G./Kußmaul, Paul/Schmitt A. Peter (Hg.) Handbuch Translation. Tübingen: Stauffenburg, 341-343.

Hönig, Hans G., 1986. Übersetzen zwischen Reflex und Reflexion - ein Modell der übersetzungsrelevanten Textanalyse. In: Snell-Hornby, Mary (Hg.) Übersetzungswissenschaft. Eine Neuorientierung. Tübingen: Francke, 230-251.

Hönig, Hans G., 1995. Konstruktives Übersetzen. Tübingen: Stauffenburg.

Holz-Mänttäri, Justa. 1984. Translatorisches Handeln. (Theorie und Methode). Helsinki: Suomalainen Tiedeakatemia. (Annales Academiae Scientiarum Fennicae B 226).

Konrad, Klaus, 1998. Kooperatives Lernen bei Studierenden: Förderung metakognitiver Selbstäußerungen und (meta)kognitive Profile. In: Unterrichtswissenschaft. Zeitschrift für Lernforschung, 67-85.

Lasswell, Harold D., 1948. Power and personality. New York: Norton.

Mackenzie, Rosemary \& Vienne, Jean 2000. Resource research strategies: A key factor in teaching translation into the non-mother tongue. In: Meta Grosman/Mira Kadric/Irena Kovačic/Mary Snell-Hornby (eds.). Translation into Non-Mother Tongues - In Professional Practice and Training. Tübingen: Stauffenburg, 125-131.

Nord, Christiane 1991.2 Textanalyse und Übersetzen. Theoretische Grundlagen, Methode und didaktische Anwendung einer übersetzungsrelevanten Textanalyse. Heidelberg: Groos.

Nord, Christiane 1998. Textanalyse: pragmatisch/funktional. In: Snell-Hornby et al. (Hg.), 350-354. 
Prunč, Erich, 2000. Translation in die Nicht-Muttersprache und Translationskultur. In: M. Grosman et. (eds.), 5-20.

Resch, Renate. 1998. Die Rolle der muttersprachlichen Kompetenz. In: Snell-Hornby et al., 343-345.

Skutnabb-Kangas, Tove, 1981. Bilingualism or Not. The Education of Minorities. (Transl. by Lars Malmberg and David Crane). Clevedon [u. a.]: Multilingual Matters (Multilingual Matters 7).

Wilss, Wolfram, 1992. Was ist Übersetzungsdidaktik? Versuch einer Faktorenanalyse. In: Lebende Sprachen 2, 56-60. 